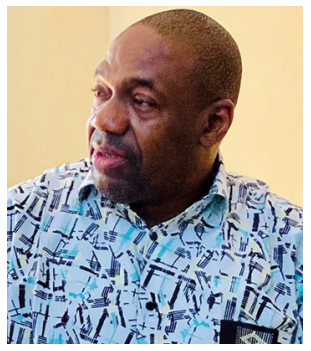

\title{
Global South cannot just live with COVID-19
}

\author{
Although the Global North is grappling with whether COVID-19 will turn endemic, \\ in Mali and other resource-poor countries 'living with COVID-19' would be devastating, \\ warns Samba Sow.
}

Credit: Dr Camilla Ducker

C OVID-19 continues to march across the globe, fuelled most recently by an Omicron variant that is more transmissible than any encountered since the onset of the global pandemic. But unlike in January 2021, when case numbers, hospitalizations and deaths all skyrocketed, the global community now has a number of vaccines at its disposal to protect populations - if not against transmission itself, then against the most-severe disease outcomes linked to infection. Therapeutic agents are also developing rapidly, so it would seem that the world has turned a corner and can begin to look forward in earnest to a time 'post-COVID-19'.

I fear, though, that this is just part of the story. And it is a story being told, largely, by countries of the Global North that have vast quantities of vaccine at their disposal, which are holding at bay the need for strict lockdowns and confinement. However, as WHO special envoy for COVID-19 in West Africa, I have seen that many countries - including my own, Mali - are playing catch-up in regard to a great number of factors that are taken for granted elsewhere. Our testing capacity remains unable to provide a comprehensive picture of the state of the epidemic in our communities. A public health policy that is based in part on regular self-testing and the isolation of cases remains, for us, a practical impossibility.

Highly vaccinated countries such France, the UK and USA may well weather their current astronomical levels of infection without the effects on their health systems being crippling. This is not the case in Mali, for example, where early 2022 indicators have shown a spike in infection and hospitalization not seen before. In a population that has not been vaccinated in anything like the numbers of other countries, this will have a devastating effect on our fragile health system. Already we have seen substantial reductions in rates of coverage for seasonal malaria control as well as routine immunizations, and these can only mean increased maternal and child mortality in the months to come.

The one area in which we may have hoped to make real progress is in vaccine equity - that is, vaccines supplies being distributed more equitably throughout the world, and not only concentrated in and reserved for the populations of high-income countries. But the COVAX initiative, which is intended to pool and distribute vaccines across lower-income countries, can only be as successful as the donations it receives. Vaccine pledges from high-income countries have, to date, been too occasional and too limited - particularly in view of the clamour for 'booster' shots in many high-income countries.

By far the most important message remains one that $I$ have repeated many times over the past few months: we need greater and more widespread vaccine equity. The rapid development of vaccines with such a high degree of safety and efficacy must rank among the greatest of human achievements in the twenty-first century to date. I fear that their distribution, however, points towards other, perhaps less-noble human characteristics. For without the bedrock of a vaccinated population, Mali and other countries in the Global South will continue to be left behind in the dynamics of COVID-19 planning and response.

While countries in the Global North look forward to 'all this being over' sooner rather than later, the pandemic still has a long way to run for the world's most disadvantaged populations. Living with COVID-19 is only possible if a large percentage of the population has some form of protection against the disease, and if health systems are able to cope with a steady number of patients with COVID-19. Neither of these preconditions currently exist in Mali, or in any number of similarly disadvantaged countries.

Even now, it seems that we as a global community have not been able to take on board one simple truth. While there is active and substantial circulation of virus, the potential for considerable loss of life and severe disease remains, as does the possibility that new and potentially even more devastating variants will emerge. The goal we should be working towards is the WHO goal of achieving 70\% COVID-19 immunization coverage by mid- 2022 .

All this may soon be over - but this will only truly be so if it is over for everybody, everywhere.

\footnotetext{
Samba O. Sow ${ }^{1,2}$ 凶

${ }^{1}$ Center for Vaccine Development, Bamako, Mali. ${ }^{2}$ National Institute for Public Health, Bamako, Mali.

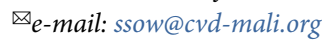

Published online: 31 January 2022 https://doi.org/10.1038/s41562-022-01296-9

Competing interests

The author declares no competing interests. 